\title{
Fc-binding antibody-recruiting molecules for cancer therapy: exploiting endogenous IgG without antigen-Fab interactions
}

\author{
Koichi Sasaki, ${ }^{\dagger}$ Minori Harada, ${ }^{\dagger}$ Yoshiki Miyashita, ${ }^{\ddagger}$ Hiroshi Tagawa, ${ }^{\ddagger}$ Akihiro Kishimura, ${ }^{\dagger},\|$,$\| Takeshi$

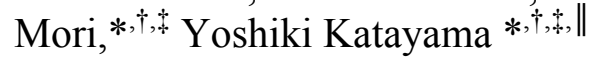 \\ ${ }^{\dagger}$ Department of Applied Chemistry, Graduate School of Engineering, Kyushu University, Fukuoka, 819-0395, Japan \\ ${ }^{\ddagger}$ Graduate School of Systems Life Sciences, Kyushu University, Fukuoka, 819-0395, Japan \\ "International Research Center for Molecular Systems, Kyushu University, Fukuoka, 819-0395, Japan
}

\begin{abstract}
Small molecules emulating the effector functions of antibodies have potential clinical benefits because of their low immunogenicity. Antibody-recruiting molecules (ARMs) are bispecific molecules designed to redirect endogenous antibodies to targets. However, endogenous antibodies show intra/inter-patient differences regarding their concentrations and affinities, limiting the potential of ARMs. We sought to address this issue using a Fc-binding peptide instead of an antigen for antibody redirection. Fc-binding ARM (Fc-ARM) targeting folate receptor- $\alpha$ (FR- $\alpha$ ) expressed on cancer cells, formed a ternary complex of Fc-ARM, FR- $\alpha$, and antibody on cancer cells. The ability of this ternary complex to activate natural killer cells was positively correlated with its Fc affinity, and did not require the Fab region. Fc-ARM hitchhiked on pooled human IgG to enhance its blood retention, and suppressed tumor growth in a mouse xenograft model of human cancer. Thus, Fc-ARM has the potential to be employed as a less immunogenic alternative to therapeutic antibodies.
\end{abstract}

\section{INTRODUCTION}

Therapeutic monoclonal antibodies (mAbs) have revolutionized the clinical outcomes for multiple cancers, including B cell lymphoma. ${ }^{1}$ However, their potential immunogenicity is one of the limitations in their clinical application. For example, IgE-mediated anaphylaxis and cytokine storms are lifethreatening side effects of $\mathrm{Ab}$ administration. ${ }^{2}$ Even fully human $\mathrm{mAb}$ (anti-tumor necrosis factor- $\alpha$ ) developed anti-drug antibodies in $17 \%$ of patients, resulting in a decreased serum concentration and reduced efficacy of the $\mathrm{mAb}{ }^{3}$ Such adverse events are thought to be attributed to the large molecular weight of mAbs and the presence of non-self amino acid sequences. $^{2}$

To address this issue, small molecules that imitate the effector functions of Abs have been developed because small molecules are thought to have low immunogenicity. ${ }^{4,5}$ Antibodyrecruiting molecule (ARM), which is composed of an antibody-binding terminus (ABT) and a target-binding terminus (TBT), is one of the promising modalities. ${ }^{6}$ ABT utilizes antigen-Fab (fragment antigen binding) interactions to form an ARM-Ab complex, and TBT plays a role in redirection of endogenous Abs to its target, such as tumor cells. Subsequently, recruited Abs activate the immune system, including immune cells, triggering elimination of the target. Although various types of TBTs have been produced to evaluate the applicability of ARMs to a broad range of targets, ${ }^{7-22}$ only three ABTs against pre-existing endogenous Abs have been investigated: galactosyl-(1-3)-galactose $(\alpha-\mathrm{Gal}),{ }^{12,13}$ rhamnose, ${ }^{21}$ and nitroarenes. ${ }^{14-16,20,22}$ The limited choice of ABTs decreases the clinical potential of ARMs because pre-existing endogenous Abs show intra/inter-patient variability in terms of characteristics such as $\mathrm{Ab}$ concentration and affinities. For example, their amounts are limited and vary among individuals (up to $2 \%$ for

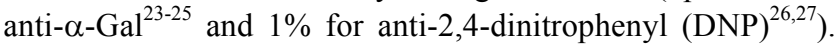
Thus, creating new ABTs to overcome the intra/inter-patient differences observed for endogenous Abs is a key challenge.

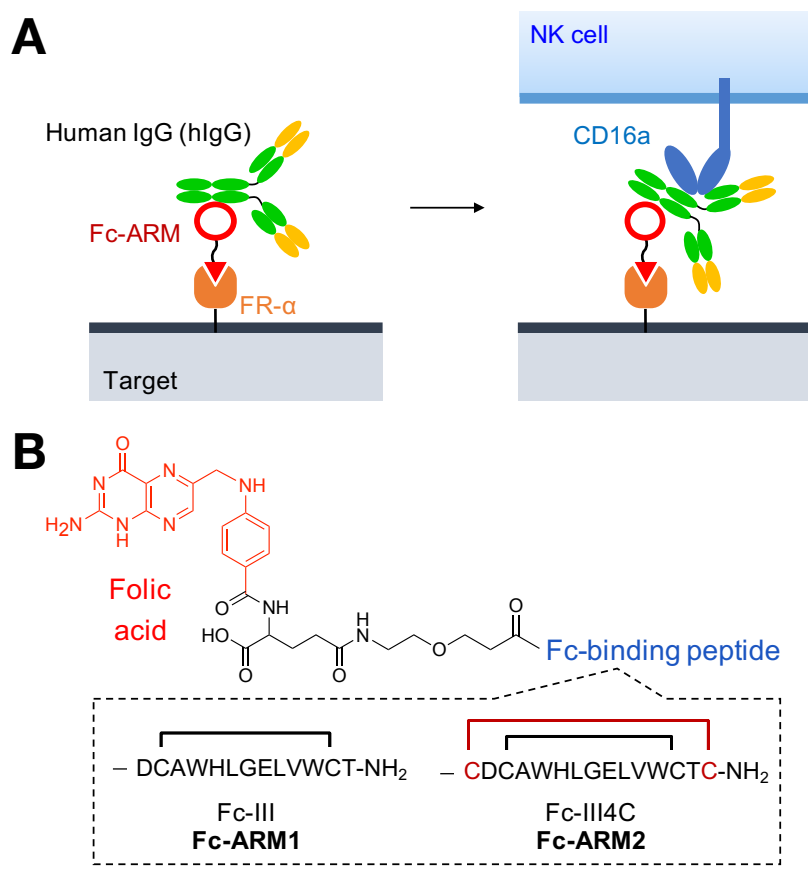

Figure 1. Schematic illustration of the Fc-ARM strategy and molecular structures of Fc-ARMs. (A) Schematic of the FcARM-mediated activation of NK cells. (B) Molecular design of Fc-ARM1 and Fc-ARM2. 
The Fc (fragment crystallizable) region of IgG is characterized by its conserved molecular structure, as well as a variety of biological functions. The Fc region operates as a module for effector functions such as antibody-dependent cell-mediated cytotoxicity (ADCC). ${ }^{28,29}$ A recent study revealed that the Fc fragment of IgG binds to herpes simplex virus 1 (HSV1) glycoprotein E and Fc-gamma receptor IIIa (FcyRIIIa)/CD16a expressed on immune cells simultaneously, resulting in ADCC against HSV1-infected cells. ${ }^{30}$ This report indicates that ADCC could be induced in a Fab-independent manner.

Previously, we have reported a new class of ARMs, namely Fc-ARMs, in which Fc-binding cyclic peptide (Fc-III) was used as an ABT and folic acid (FA) was used as a TBT to target tumor cells that overexpress folate receptor- $\alpha(\mathrm{FR}-\alpha){ }^{31}$ Using Fc-ARM, we sought to exploit the majority of endogenous IgGs to eliminate malignant cells independently of antigen-Fab interactions (Figure 1A). We previously demonstrated that a ternary complex of Fc-ARM, FR- $\alpha$, and human IgG (hIgG) can be formed on the surface of cancer cells. ${ }^{31}$ This ternary complex, however, did not mediate ADCC in the previous experimental settings. Given that $\mathrm{ADCC}$ is regulated by the overall affinity of $\mathrm{Ab}$ to its cognate antigen ${ }^{32}$ and $\mathrm{CD} 16 \mathrm{a}^{33}$ we hypothesized that replacement of the Fc-III peptide with recently reported peptide, $\mathrm{Fc}-\mathrm{III} 4 \mathrm{C}$, which has higher affinity for IgG-Fc, ${ }^{34}$ would strengthen the ternary complex to activate effector cells such as natural killer (NK) cells for target cell destruction. We evaluated the formation of the ternary com- plex, as well as its anti-tumor efficacy, both in vitro and in vivo.

\section{RESULTS AND DISCUSSION}

We used an Fc-III peptide or an Fc-III4C peptide $\left(\mathrm{K}_{\mathrm{d}}\right.$ against $\mathrm{hIgG}-\mathrm{Fc}$ reported to be $70 \mathrm{nM}$ and $2.5 \mathrm{nM}$, respectively) ${ }^{34}$ as an ABT. FA was selected as a TBT. FA has high affinity to FR- $\alpha\left(\mathrm{K}_{\mathrm{d}}=0.19 \mathrm{nM}\right)^{35}$ and is therefore used for targeted drug delivery and imaging of FR- $\alpha$-overexpressing cancers. ${ }^{36}$ The ABT and the TBT were connected with a hexaethylene glycol linker, and two types of Fc-ARMs were synthesized, namely Fc-ARM1 and Fc-ARM2 (Figure 1B). Fc-ARM2 is a new molecule synthesized in this report, whereas Fc-ARM1 was used in our previous study. ${ }^{31}$ The Fc-ARMs were characterized by reverse phase high performance liquid chromatography (RP-HPLC, Figure S1) and matrix assisted laser desorption/ionization-time-of-flight mass spectrometry (MALDITOF MS, Figure S2). First, we used surface plasmon resonance (SPR) to measure the affinities between Fc-ARMs and hIgG1. We functionalized SPR chips with Herceptin and flowed Fc-ARMs over the chip surface. The results showed that Fc-ARM2 had higher affinity to hIgG1 compared with FcARM1 (Figure 2A-C). The $\mathrm{K}_{\mathrm{d}}$ values were $25.0 \mathrm{nM}$ for FcARM1 and $6.1 \mathrm{nM}$ for Fc-ARM2. Next, we verified whether the ternary complex of FR- $\alpha$, Fc-ARM2, and hIgG could be formed similarly to Fc-ARM1. ${ }^{31}$ Fluorescent microscopy revealed that FITC-labeled hIgG (hIgG-FITC) successfully bound to IGROV-1 cells in the presence of Fc-ARM2 (Figure S3).
A

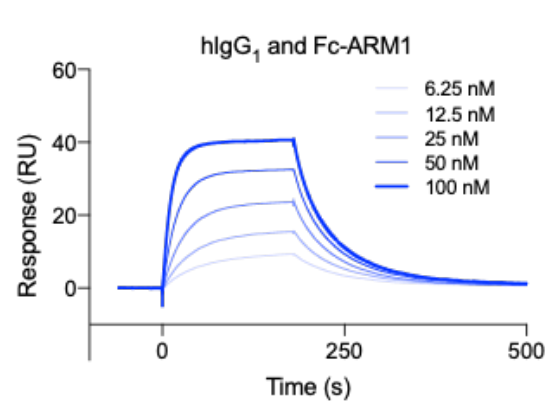

D

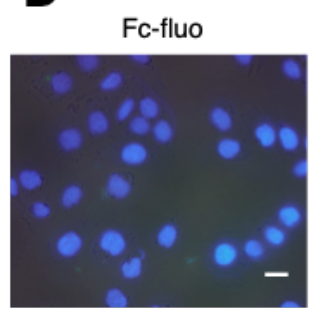

B

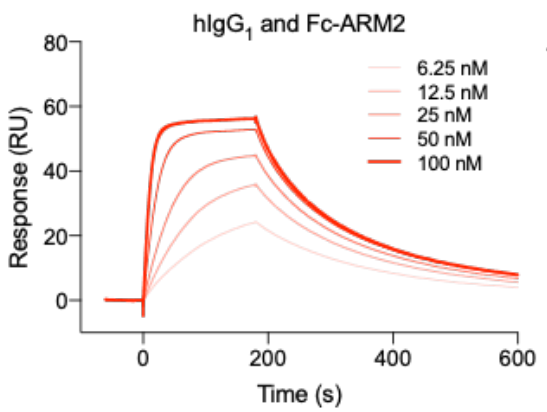

\begin{tabular}{l|c|c} 
& Fc-ARM1 & Fc-ARM2 \\
\hline $\mathrm{k}_{\text {on }}(/ \mathrm{Ms})$ & $6.1 \times 10^{5}$ & $1.6 \times 10^{6}$ \\
$\mathrm{k}_{\text {off }}(/ \mathrm{s})$ & $1.6 \times 10^{-2}$ & $9.9 \times 10^{-3}$ \\
$\mathrm{~K}_{\mathrm{d}}(\mathrm{M})$ & $2.5 \times 10^{-8}$ & $6.1 \times 10^{-9}$
\end{tabular}

E

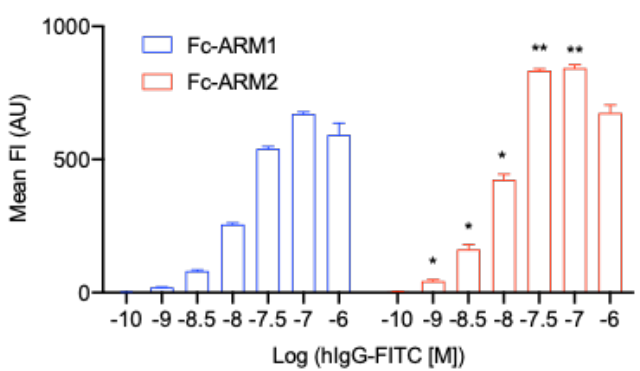

Figure 2. Fc-ARM2 shows high affinity for IgG-Fc and recruits antibody to FR- $\alpha^{+}$cells. (A-C) Dissociation constants of (A) FcARM1 and (B) Fc-ARM2 to human IgG1 were determined by SPR measurements. (C) $\mathrm{k}_{\text {on }}$, $\mathrm{k}_{\text {off }}$, and $\mathrm{K}_{\mathrm{d}}$ values for Fc-ARMs are summarized. (D) IGROV-1 cells were seeded and incubated overnight. Fc-fluorescein (500 nM), Fc-fluorescein + Fc-ARM2 (100 $\mathrm{nM})$, or Fc-fluorescein + Fc-ARM2 + excess FA $(100 \mu \mathrm{M})$ were added. After washing, cells were stained with Hoechst 33342. Scale bar $=20 \mu \mathrm{m}$. Representative pictures are shown. (E) IGROV-1 cells were treated with $10 \mathrm{nM}$ of Fc-ARMs and increasing concentrations of IgG-FITC. The mean fluorescence intensity (FI) derived from the ternary complex was quantified by flow cytometry $(\mathrm{n}=3$, mean \pm SEM). Two experimental repeats were performed. Statistical analyses were carried out using a two-tailed Welch's $t$ test. $* \mathrm{p}<0.05 ; * * \mathrm{p}<0.01$. 
Excess FA diminished hIgG-derived fluorescence. Furthermore, the papain-digested Fc fragment of anti-CD20 mAb (Ofatumumab, Figure S4) labeled with fluorescein (Fc-fluo) was also recruited on IGROV-1 cells by Fc-ARM2 (Figure 2D). These results demonstrated that ternary complex formation is driven by the bispecific affinities of Fc-ARM2 to FR- $\alpha$ and the Fc region of hIgG. Next, we conducted a quantitative evaluation of ternary complex formation. Considering that $10 \mathrm{nM}$ of Fc-ARM1 saturated the fluorescent intensity derived from the ternary complex (Figure S5), $10 \mathrm{nM}$ of FcARM (Fc-ARM1 or Fc-ARM2) and increasing concentrations of IgG-FITC were added to IGROV-1 cells. Fc-ARM2 showed significantly higher mean fluorescence intensity (FI) derived from the ternary complex compared with Fc-ARM1 (Figure 2E). Notably, $1 \mu \mathrm{M}$ of IgG-FITC reduced the mean FI, a typical characteristic of ternary complex formation. ${ }^{37}$ These data suggested that Fc-ARM2 possesses improved affinity to IgG-Fc, enabling more efficient formation of the ternary complex.

Next, we evaluated whether formation of the ternary complex can activate NK cells and induce target cell destruction. Importantly, we previously reported that the binding sites of Fc-binding peptide and CD16a to the Fc region of the antibody do not overlap, ${ }^{31}$ rationalizing the feasibility of CD16amediated immune cell activation by the ternary complex. Ofatumumab was used as a source of Ab since its ADCC capacity against $\mathrm{CD} 20^{+}$cells was certified, ${ }^{38}$ and IGROV-1 cells do not express CD20. IGROV-1 cells were co-cultured with human NK cells (KHYG-1/CD16a-158V) ${ }^{39}$ for $16 \mathrm{~h}$ in the presence of Fc-ARM $(10 \mathrm{nM})$ and anti-CD20 mAb $(100 \mathrm{nM})$, then lactose dehydrogenase (LDH) released from lysed cells was quantified.
A

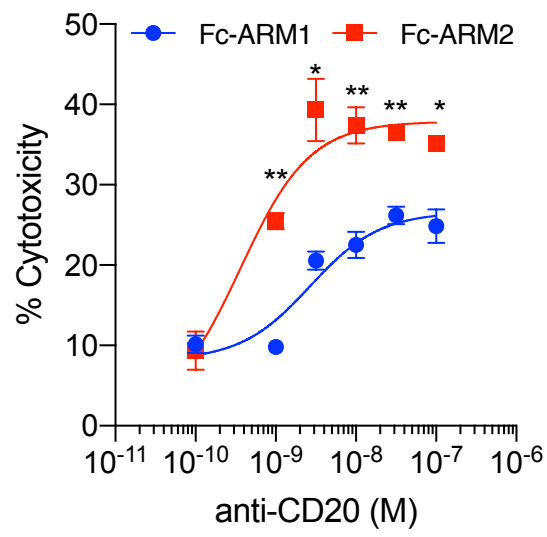

B

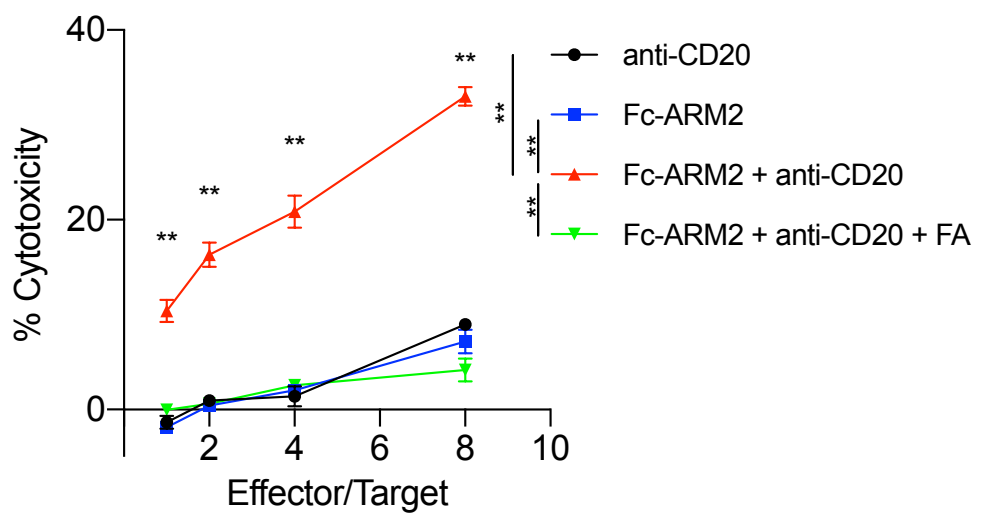

C

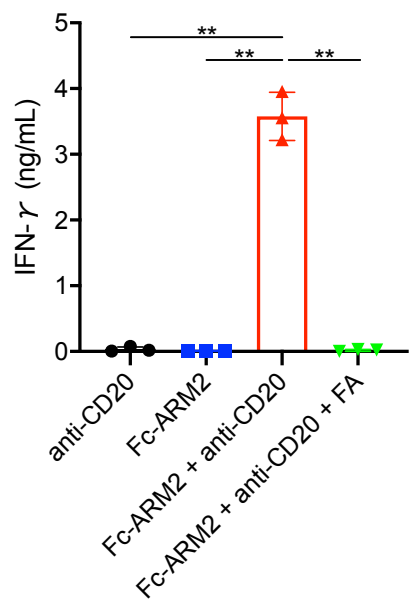

D

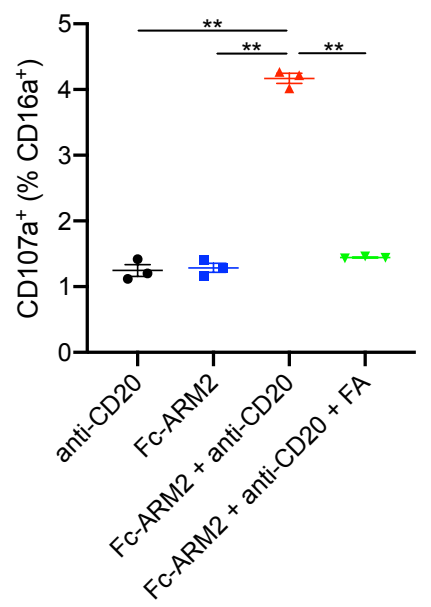

E

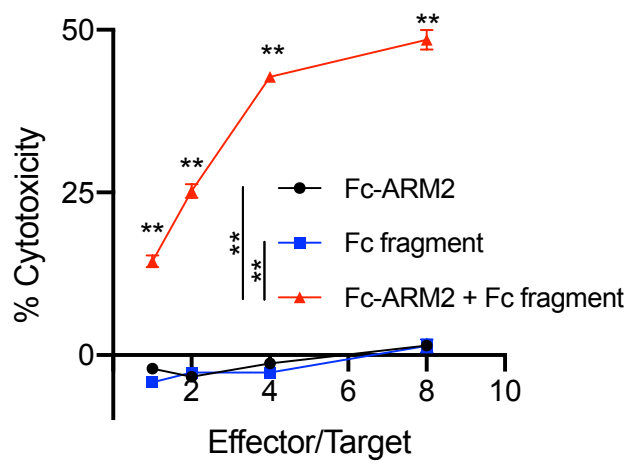

Figure 3. Fc-ARM2 effectively recruits hIgG1 and the Fc fragment onto the FR- $\alpha^{+}$cell surface and activates NK cells. (A) 5000 cells/well of IGROV-1 cells were co-cultured with 40000 cells/well of KHYG-1/CD16a-158V in the presence of Fc-ARM (10 nM) and various concentrations of anti-CD20 for $16 \mathrm{~h}$. ADCC activity was quantified by an LDH assay $(\mathrm{n}=3, \mathrm{mean} \pm \mathrm{SEM})$. (B) 5000 cells/well of IGROV-1 cells were treated with anti-CD20 (100 nM), Fc-ARM (10 nM), or Fc-ARM2 + anti-CD20, or Fc-ARM2 + anti-CD20 + FA $(1 \mu \mathrm{M})$ and co-cultured with $5000-40000$ cells/well of KHYG-1/CD16a-158V for $16 \mathrm{~h}$. $(\mathrm{n}=3$, mean \pm SEM). $(\mathrm{C})$ After co-culturing of IGROV-1 cells and KHYG-1/CD16a-158V, culture supernatants were collected for human IFN- $\gamma$ detection by ELISA (E/T $=8$, mean \pm SEM). (D) Surface mobilization of CD107a on KHYG-1/CD16a-158V cells was evaluated after 6 h of stimulation by flow cytometry $(\mathrm{E} / \mathrm{T}=1$, mean $\pm \mathrm{SEM})$. (E) An ADCC assay was performed using the Fc fragment of anti-CD20 $\mathrm{mAb}(\mathrm{n}=3$, mean $\pm \mathrm{SEM})$. Two experimental repeats were performed. Statistical analyses were carried out using (A) a two-tailed Welch's $t$ test, or (B-E) one-way ANOVA with Tukey's multiple comparison test. ${ }^{*} \mathrm{p}<0.05 ;{ }^{*} \mathrm{p}<0.01$. 
When compared with Fc-ARM1, Fc-ARM2 showed enhanced cytotoxicity along with increased concentrations of anti-CD20 (Figure 3A). Fc-ARM2 in combination with antiCD20 showed clear target cell killing in an effector/target ratio-dependent manner, whereas anti-CD20 or Fc-ARM2 did not (Figure 3B). Cytotoxicity was decreased to basal levels by excess FA (Figure 3B). Target cell killing was not observed when A549 lung cancer cells (FR- $\left.\alpha^{-}\right)$were used as the target cells (Figure S6), consistent with our previous observation that the ternary complex was not formed on A549 cells. ${ }^{31}$ Upon stimulation by the ternary complex, NK cells showed secretion of interferon- $\gamma($ IFN- $\gamma$ ) (Figure 3C) and surface mobilization of CD107a (activation marker of NK cells, ${ }^{40}$ Figure 3D and S7), further demonstrating that the ternary complex can activate NK cells. Notably, the Fc-fragment of anti-CD20 mAb also induced cytotoxicity when used in combination with FcARM2 (Figure 3E). Taken together, these results demonstrat- ed that the ternary complex of FR- $\alpha$, Fc-ARM, and anti-CD20 hIgG1 is capable of activating NK cells for target cell destruction without antigen-Fab interactions.

We next conducted in vitro assays over shorter co-culture time periods to investigate whether or not the affinity of Fcbinding peptide determines the immune-stimulatory activity of the ternary complex. Fc-ARM1 in combination with antiCD20 (Fc-ARM1 + anti-CD20) did not show cytotoxicity after $4 \mathrm{~h}$ of co-culture (Figure S8A), in accordance with our previous report. ${ }^{31}$ Fc-ARM2 showed higher cytotoxicity compared with Fc-ARM1 in combination with anti-CD20 after 6, 8, and $16 \mathrm{~h}$ of co-culture (Figure S8 B-D). Fc-ARM2 + anti-CD20 induced significantly higher amount of IFN- $\gamma$ secretion than Fc-ARM1 + anti-CD20 after 16 h of co-culture (Figure S8E). These results clearly demonstrated that the Fc-affinity of FcARM is crucial for efficient activation of NK cells by the ternary complex.
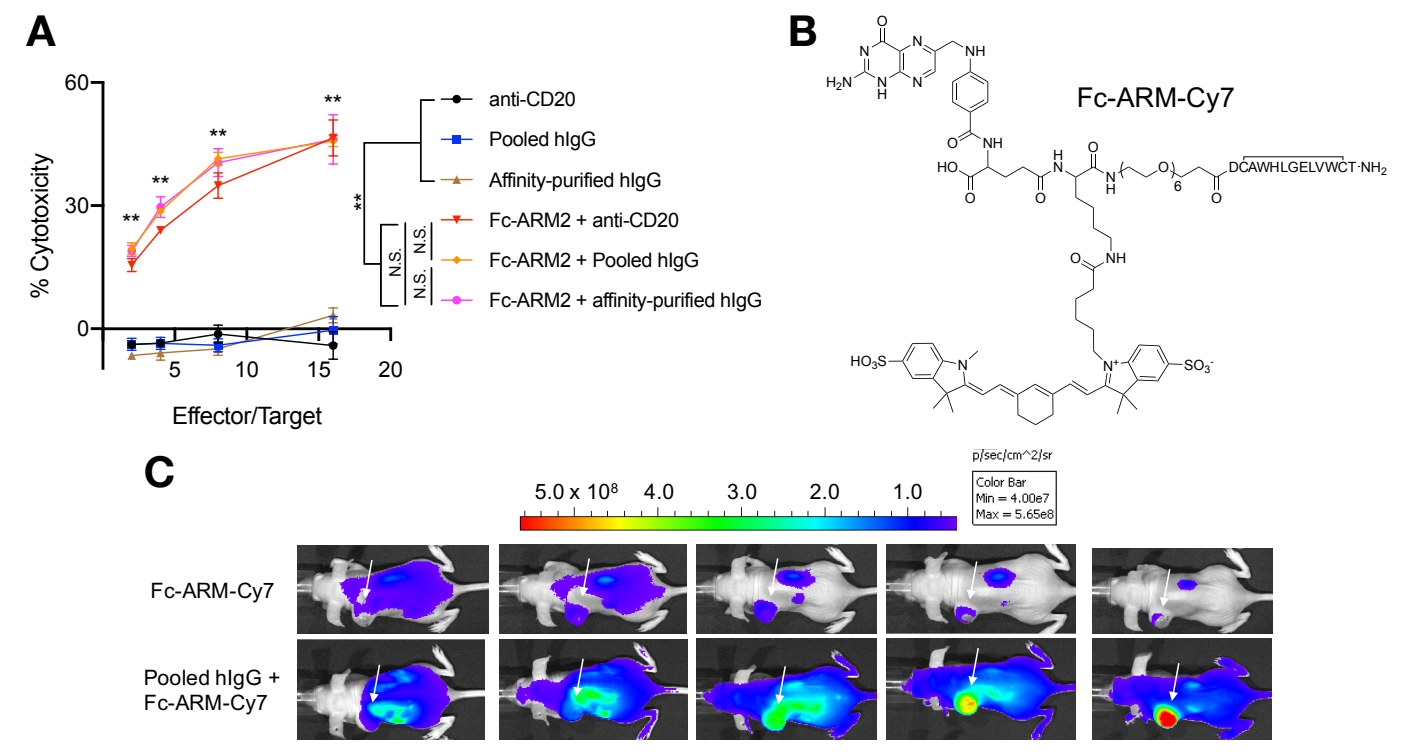

$1 \mathrm{~h}$

$3 \mathrm{~h}$

$6 \mathrm{~h}$

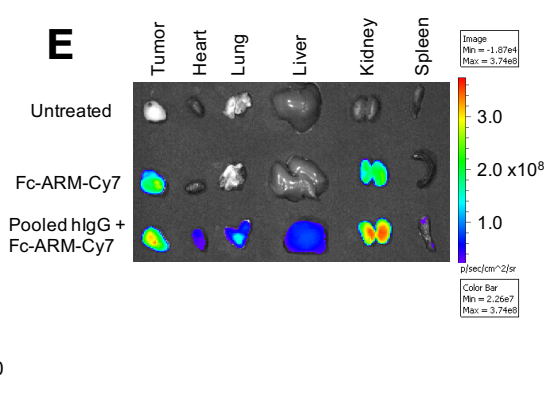

p/sec/cm^2/sr Color Bar
Min $=4.00 \mathrm{e}$
$\operatorname{Max}=5.6598$
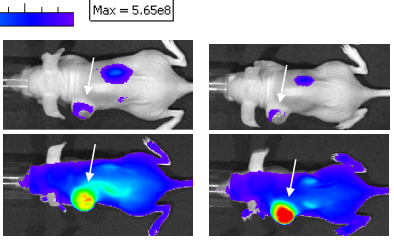

$12 \mathrm{~h}$

$24 \mathrm{~h}$

$\mathbf{F}$

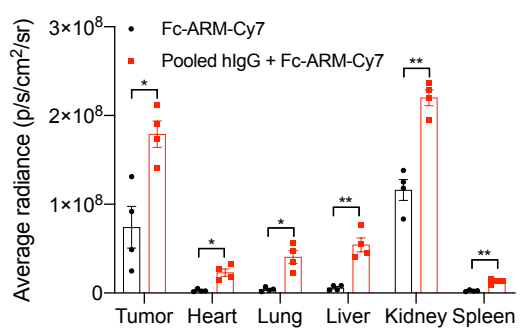

Figure 4. Fc-ARM interacts with pooled hIgG in vivo to enhance its blood retention time and tumor accumulation. (A) Two different products of IgG from human sera were compared with anti-CD20 mAb. hIgG (100 nM) and Fc-ARM2 (10 nM) were added into 5000 cells/well of IGROV-1 cells. 10000-80000 cells/well of KHYG-1/CD16a-158V cells were added and incubated for 16 h. LDH released from lysed cells was quantified $\left(n=3\right.$, mean \pm SEM). (B) The molecular structure of Fc-ARM-Cy7. $(\mathrm{C}-\mathrm{F}) 1 \times 10^{6}$ of IGROV-1 cells were inoculated into BALB/c nu/nu mice. When the tumor volume reached about $300 \mathrm{~mm}^{3}$, mice received $20 \mathrm{mg}$ of pooled hIgG intraperitoneally. After $2 \mathrm{~h}$, mice received $5 \mathrm{nmol}$ of Fc-ARM-Cy 7 intraperitoneally. (C) In vivo fluorescence imaging of Fc-ARM-Cy7 at different time points was performed using the IVIS Lumina II. Representative images from four mice in each group are shown. (D) Time-course quantification of the fluorescent signals from tumors $(\mathrm{n}=4$, mean $\pm \mathrm{SEM})$. (E) At $24 \mathrm{~h}$ after FcARM-Cy7 injection, organs were harvested and imaged ex vivo. A representative image of the organs is shown ( $\mathrm{n}=4$ per group). (F) Fluorescent signals were quantified (mean \pm SEM). Two experimental repeats were performed. Statistical analyses were carried using (A) one-way ANOVA with Tukey's multiple comparison test or (D, F) a two-tailed Welch's t test. *p $<0.05$; **p $<0.01$; N.S. = not significant. 
Previously, we reported that Fc-ARM1 inhibits the ADCC activity of anti-epidermal growth factor receptor (EGFR) $\mathrm{mAb}$ against IGROV-1 cells (EGFR/FR- $\alpha$ double positive). ${ }^{31}$ To investigate the mechanism of action, we first compared the amount of EGFR/anti-EGFR binary complex with that of the ternary complex formed on IGROV-1 cells using flow cytometry. IGROV-1 cells were incubated with fluoresceinlabeled anti-EGFR mAb (Cetuximab). The results showed that $5 \mathrm{nM}$ of anti-EGFR mAb was sufficient to saturate binary complex formation (Figure S9A). Furthermore, $10 \mathrm{nM}$ of FcARM1 substantially increased the mean FI, showing that the capacity to form the ternary complex on IGROV-1 cells is greater than that of the binary complex. Next, we evaluated the ADCC activity of both complexes (Figure S9B). The binary complex of EGFR/anti-EGFR mAb showed stronger ADCC activity compared with the ternary complex (Fc-ARM2 + antiCD20). Interestingly, co-existence of the binary complex and the ternary complex (Fc-ARM2 + anti-EGFR) resulted in similar ADCC activity compared with the ternary complex (FcARM2 + anti-CD20). These results indicated that the ternary complex activates NK cells, but also works as a competitive inhibitor of CD16a against stronger agonists (in this case, the binary complex of EGFR/anti-EGFR mAb). We do not discuss the potential contribution of the quaternary complex of FR- $\alpha$, Fc-ARM2, anti-EGFR, and EGFR in this manuscript because we are currently unable to isolate the quaternary complex.

Based on the finding that the ternary complex has the ability to activate NK cells for tumor cell killing, we sought to test the therapeutic potential of the Fc-ARM strategy. We investigated whether Fc-ARM2 was capable of inducing ADCC with a mixture of hIgG subclasses and found that Fc-ARM2 + pooled hIgG (Venoglobulin) and Fc-ARM2 + affinity-purified hIgG from sera clearly induced ADCC against IGROV-1, whereas hIgG products alone did not (Figure 4A). The levels of ADCC induced by these two conditions were similar to that induced by Fc-ARM2 + anti-CD20, indicating that endogenous hIgGs could potentially be used as a source of Abs for immune cell activation that would be as efficient as hIgG1. Next, we prepared a derivative of Fc-ARM1 labeled with sulfo-cyanine 7 (Fc-ARM-Cy7, Figure 4B and S10) to investigate the interactions between Fc-ARM and IgGs in vivo. Since FcARM2 did not induce ADCC in combination with mouse IgG (mIgG) due to an inability to bind to $\mathrm{mIgG}$ (Figure S11), we pre-injected tumor-bearing mice with hIgG to achieve ternary complex formation in vivo. Injected hIgG is expected to remain in the blood circulation for exceptionally long time as a macromolecule because it cross-reacts with mouse neonatal Fc receptor $(\mathrm{FcRn}),{ }^{41}$ which is responsible for salvaging Abs from lysosomal degradation. Thus, we hypothesized that preinjection of hIgGs into mice enhances the blood circulation time and the tumor accumulation of Fc-ARM. Based on a previous study, $20 \mathrm{mg}$ of pooled hIgG was injected per mouse to mimic the blood concentration of IgG in humans. ${ }^{42} \mathrm{Next}, 2 \mathrm{~h}$ after intraperitoneal injection of pooled hIgG, IGROV-1 tumor-bearing $\mathrm{BALB} / \mathrm{c} \mathrm{nu} / \mathrm{nu}$ mice were injected intraperitoneally with $5 \mathrm{nmol}$ of Fc-ARM-Cy7. In vivo time-course imaging showed that pooled hIgG significantly improved the blood retention time and tumor-targeted delivery of Fc-ARM-Cy7 (Figure 4C, D).
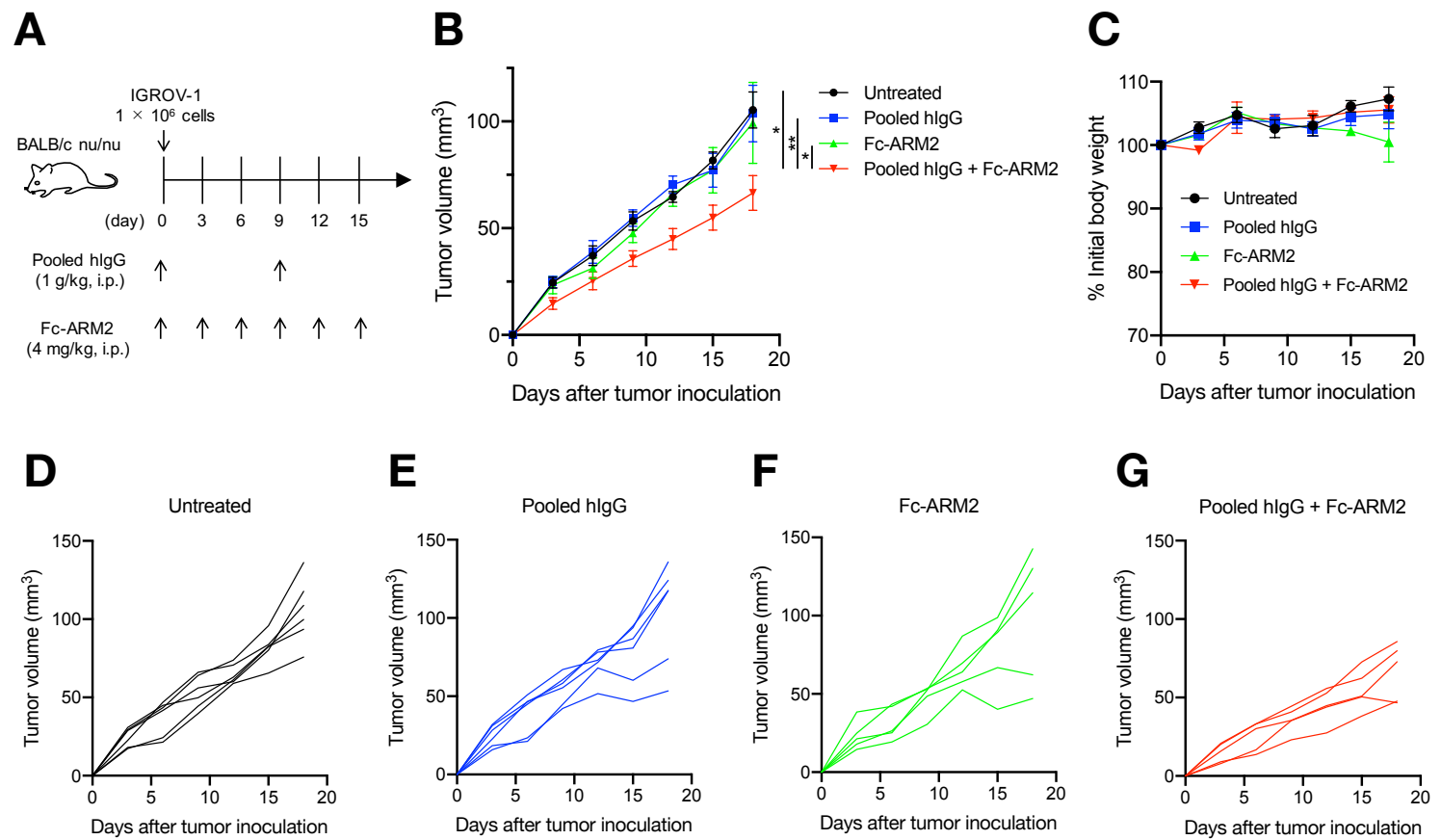

Figure 5. Fc-ARM2 suppresses IGROV-1 tumor growth in combination with pooled hIgG. (A) The treatment schedule for IGROV-1 tumors is shown. Mice were injected intraperitoneally with $1 \mathrm{~g} / \mathrm{kg}$ of pooled hIgG. Then, after $2 \mathrm{~h}$, mice were injected intraperitoneally with $4 \mathrm{mg} / \mathrm{kg}$ of Fc-ARM2. (B) The graph depicts the tumor volume up until day 18 ( $\mathrm{n}=6$ for untreated and pooled hIgG, $\mathrm{n}=5$ for Fc-ARM2 and pooled hIgG + Fc-ARM2, mean \pm SEM). (C) Body weight changes in the mice during treatment (mean \pm SEM). (D-G) Individual growth curves for the IGROV-1 tumors shown in Figure 6B. Two experimental repeats were performed. Statistical analyses were carried out using one-way ANOVA with Tukey's multiple comparison test (on day 12). $* \mathrm{p}<0.05 ; * *_{\mathrm{p}}<0.01$. 
Ex vivo imaging at $24 \mathrm{~h}$ after Fc-ARM-Cy7 injection showed that significantly higher amounts of Fc-ARM-Cy7 remained in a range of dissected organs in mice that received prior injection of pooled hIgG (Figure 4E, F). These data suggested that Fc-ARM interacts with hIgGs and harnesses their pharmacokinetics, resulting in an enhanced blood circulation time and accumulation in tumors.

Finally, we tested the anti-tumor efficacy of the ternary complex in vivo. Since there is crosstalk between $\mathrm{hIgG}$ and mouse effector cells, including mouse NK cells, ${ }^{43}$ we hypothesized that the ternary complex of Fc-ARM2, FR- $\alpha$, and hIgG activates mouse NK cells and inhibits tumor growth in vivo. IGROV-1-bearing BALB/c nu/nu mice were used. A summary of the treatment schedule is shown in Figure 5A. Given that hIgG1 shows a blood half-life of about 10 days in mice, ${ }^{44}$ pooled hIgG $(1 \mathrm{~g} / \mathrm{kg})$ was injected intraperitoneally into mice 0 and 9 days after tumor inoculation. Fc-ARM2 (4 mg/ $\mathrm{kg})$ was intraperitoneally administered to mice $0,3,6,9,12$, and 15 days after tumor inoculation. On days 0 and 9 , mice received Fc-ARM2 $2 \mathrm{~h}$ after hIgG injection. Fc-ARM2 + pooled hIgG significantly suppressed tumor growth compared with all of the other groups (Figure 5B, 5D-G). No abnormal weight loss was observed during the treatment (Figure 5C). These data demonstrated the anti-tumor efficacy of the ternary complex in vivo.

Small molecules that emulate the effector functions of Abs can potentially balance therapeutic effects with reduced immunogenicity. As one such immunotherapeutic, ARMs bridge pre-existing endogenous Abs with target cells, resulting in immune cell activation through Fc receptors such as CD16a. ${ }^{6}$ Pre-existing endogenous Abs, however, suffer from heterogeneous characteristics and are in short supply in the circulation. $^{23-27}$ Immunization of patients with ABTs may help to manage the various characteristics of anti-ABT endogenous $\mathrm{Abs},{ }^{6,9,17,20,22}$ but increases the complexity of treatment procedures, and may also confer additional side effects. Here, we report the first ARM that is capable of immune-mediated target cell destruction independent of antigen-Fab interactions. Using an Fc-binding peptide (Fc-III or Fc-III4C) as an ABT enables Fc-ARM to access at least hIgG1 and $\mathrm{hIgG} 2^{45}$ with constant affinity. These two IgG subclasses account for more than $80 \%$ of the total IgG in humans, ${ }^{46,47}$ suggesting that at least 10 times higher amounts of $\mathrm{IgG}$ can be utilized by FcARM compared with previously reported ARMs, which have focused on endogenous Abs against $\alpha-\mathrm{Gal}^{12,13}$ or nitroarenes. $^{14-16,20,22}$

First, we showed that Fc-III4C peptide as an ABT outperforms Fc-III peptide. NK cell activation by recruited Abs was confirmed by ADCC, IFN- $\gamma$ secretion, and NK cell surface mobilization of CD107a. The positive correlation between the Fc-affinity of Fc-ARM and ADCC activity was in accordance with previous literature that reported the increased ADCC efficacy of a mAb with higher affinity to its target. ${ }^{32}$ ADCC activity was also correlated with the concentration of antiCD20 mAbs. Moreover, the Fc fragment of IgG Abs induced ADCC in combination with Fc-ARM2. These observations clearly showed that the ternary complex of Fc-ARM, FR- $\alpha$, and $\mathrm{hIgG}$ induces ADCC. Another important finding was that Fc-ARM2 + pooled hIgG from serum induced a similar level of ADCC compared with Fc-ARM2 + anti-CD20 hIgG1 mAb. This result indicated that IgG1 is a crucial subclass for induc- ing ADCC in the Fc-ARM strategy, considering that hIgG1 is the main component of pooled hIgG from sera.

Small molecules are readily removed from systemic circulation through renal filtration. As we showed in mice, Fc-ARM hitchhikes on $\mathrm{hIgG}$ to avoid this phenomenon, thereby resulting in enhanced blood retention time and tumor accumulation. Finally, we demonstrated that combination therapy with pooled hIgG and Fc-ARM2 reduces FR- $\alpha^{+}$tumor growth in T cell-deficient nude mice. Whereas, monotherapy with either Fc-ARM2 or pooled hIgG did not show anti-tumor efficacy, indicating that ternary complex formation is required for antitumor responses. The highest concentration of hIgG1 among all hIgG subclasses in blood is encouraging for our molecular design, given that hIgG1 is the most potent hIgG subclass for ADCC induction ${ }^{48}$ and the most commonly used hIgG subclass for cancer therapy in the clinic. ${ }^{49}$

One potential limitation of our strategy is that the anti-tumor efficacy of Fc-ARM may be influenced by the glycosylation patterns of endogenous IgGs in each patient. In fact, an $\mathrm{Ab}$ with afucosylation at the binding site with CD16a showed enhanced ADCC activity compared with a fucosylated Ab. ${ }^{50}$ We suggest that recently reported strategies that enable in situ modification of glycans on glycoproteins ${ }^{51,52}$ may be useful for the quality management of endogenous Abs for the Fc-ARM strategy.

\section{CONCLUSION}

In conclusion, Fc-ARM2 showed high affinity to the Fc region of IgG and formed a ternary complex of Fc-ARM2, FR- $\alpha$, and hIgG. As a consequence, recruited Abs suppressed the growth of IGROV-1 human ovarian adenocarcinoma in a mouse xenograft model. Fc-affinity would provide ARM with sufficient, robust, opportunities to redirect endogenous IgG to malignant cells. Therefore, the Fc-ARM strategy may lead to the reemergence of ARMs as one of the promising modalities of immunotherapy for cancers.

\section{ASSOCIATED CONTENT}

Supporting Information

The Supporting Information is available free of charge.

Methods, supplementary figures and supplementary references (PDF)

\section{AUTHOR INFORMATION}

Corresponding Authors

*E-mail: mori.takeshi.880@m.kyushu-u.ac.jp

*E-mail: ykatatcm@mail.cstm.kyushu-u.ac.jp

ORCID

Koichi Sasaki: 0000-0001-9966-957X

Akihiro Kishimura: 0000-0002-0503-1418

Takeshi Mori: 0000-0002-1821-5427

Yoshiki Katayama: 0000-0002-4957-6241

\section{Author Contributions}

K.S., T.M., and Y.K. designed the project. K.S., M.H., Y.M., and H.T. performed the experiments. K.S., M.H., H.T., and T.M. analyzed the data. K.S., T.M., A. K., and Y.K. wrote the manuscript. All authors read and approved the manuscript. 
Notes

Authors declare no competing financial interests.

\section{ACKNOWLEDGMENT}

K.S. was supported by Research Fellowship for Young Scientists (JSPS, 17J05032) and Advanced Graduate Course on Molecular Systems for Devices (Kyushu University). We thank Dr. Y. Yonemitsu and Dr. Y. Harada (Kyushu University) for fruitful discussions. We thank Center for Advanced Instrumental and Educational Supports at Kyushu University, Dr. M. Goto and Dr. N. Kamiya (Kyushu University) for flow cytometer. We thank Dr. K. Muguruma, (Tokyo University of Pharmacy and Life Sciences), Dr. S. Kishimoto (Kagoshima University), and Dr. Y. Ito (Kagoshima University) for assistances with SPR experiments. We thank Dr. K. Fox from Edanz Group (www.edanzediting.com/ac) for editing a draft of this manuscript.

\section{REFERENCES}

(1) Coiffier, B., Lepage, E., Briere, J., Herbrecht, R., Tilly, H., Bouabdallah, R., Morel, P., Van Den Neste, E., Salles, G., Gaulard, P., et al. (2002). CHOP chemotherapy plus rituximab compared with CHOP alone in elderly patients with diffuse large-Bcell lymphoma. N. Engl. J. Med 346, 235-242.

(2) Hansel, T.T., Kropshofer, H., Singer, T., Mitchell, J.A., and George, A.J. (2010). The safety and side effects of monoclonal antibodies. Nat. Rev. Drug Discov. 9, 325-338.

(3) Bartelds, G.M., Wijbrandts, C.A., Nurmohamed, M.T., Stapel, S., Lems, W.F., Aarden, L., Dijkmans, B.A., Tak, P.P., and Wolbink, G.J. (2007). Clinical response to adalimumab: relationship to anti-adalimumab antibodies and serum adalimumab concentrations in rheumatoid arthritis. Ann. Rheum. Dis. 66, 921-926.

(4) Imai, K., and Takaoka, A. (2006). Comparing antibody and small-molecule therapies for cancer. Nat. Rev. Cancer 6, 714727.

(5) Rader, C. (2015). Chemical biology: how to minimalize antibodies. Nature 518, 38-39.

(6) McEnaney, P.J., Parker, C.G., Zhang, A.X., and Spiegel, D.A. (2012). Antibody-recruiting molecules: an emerging paradigm for engaging immune function in treating human disease. ACS Chem. Biol. 7, 1139-1151.

(7) Bertozzi, C.R., and Bednarski, M.D. (1992). A receptormediated immune response using synthetic glycoconjugates. J. Am. Chem. Soc. 114, 5543-5546.

(8) Rader, C., Sinha, S.C., Popkov, M., Lerner, R.A., and Barbas III, C.F. (2003). Chemically programmed monoclonal antibodies for cancer therapy: adaptor immunotherapy based on a covalent antibody catalyst. Proc. Natl. Acad. Sci. U.S.A. 100, 5396-5400.

(9) Lu, Y., Sega, E., and Low, P.S. (2005). Folate receptor-targeted immunotherapy: induction of humoral and cellular immunity against hapten-decorated cancer cells. Int. J. Cancer 116, 710719.

(10) Krishnamurthy, V.M., Quinton, L.J., Estroff, L.A., Metallo, S.J., Isaacs, J.M., Mizgerd, J.P., and Whitesides, G.M. (2006). Promotion of opsonization by antibodies and phagocytosis of Grampositive bacteria by a bifunctional polyacrylamide. Biomaterials 27, 3663-3674.

(11) Doppalapudi, V.R., Tryder, N., Li, L., Aja, T., Griffith, D., Liao, F.-F., Roxas, G., Ramprasad, M.P., Bradshaw, C., and Barbas III, C.F. (2007). Chemically programmed antibodies: endothelin receptor targeting CovX-Bodies. Bioorg. Med. Chem. Lett. 17, 501-506.

(12) Carlson, C.B., Mowery, P., Owen, R.M., Dykhuizen, E.C., and Kiessling, L.L. (2007). Selective tumor cell targeting using lowaffinity, multivalent interactions. ACS Chem. Biol. 2, 119-127.

(13) Perdomo, M.F., Levi, M., Sällberg, M., and Vahlne, A. (2008). Neutralization of HIV-1 by redirection of natural antibodies. Proc. Natl. Acad. Sci. U.S.A. 105, 12515-12520.
(14) O’Reilly, M.K., Collins, B.E., Han, S., Liao, L., Rillahan, C., Kitov, P.I., Bundle, D.R., and Paulson, J.C. (2008). Bifunctional $\mathrm{CD} 22$ ligands use multimeric immunoglobulins as protein scaffolds in assembly of immune complexes on B cells. J. Am. Chem. Soc. 130, 7736-7745.

(15) Parker, C.G., Domaoal, R.A., Anderson, K.S., and Spiegel, D.A. (2009). An antibody-recruiting small molecule that targets HIV gp120. J. Am. Chem. Soc. 131, 16392-16394.

(16) Murelli, R.P., Zhang, A.X., Michel, J., Jorgensen, W.L., and Spiegel, D.A. (2009). Chemical control over immune recognition: a class of antibody-recruiting small molecules that target prostate cancer. J. Am. Chem. Soc. 131, 17090-17092.

(17) Popkov, M., Gonzalez, B., Sinha, S.C., and Barbas III, C.F. (2009). Instant immunity through chemically programmable vaccination and covalent self-assembly. Proc. Natl. Acad. Sci. U.S.A. 106, 4378-4383.

(18) Gavrilyuk, J., Uehara, H., Otsubo, N., Hessell, A., Burton, D.R., and Barbas III, C.F. (2010). Potent inhibition of HIV-1 entry with a chemically programmed antibody aided by an efficient organocatalytic synthesis. ChemBioChem 11, 2113-2118.

(19) Wuellner, U., Gavrilyuk, J.I., and Barbas III, C.F. (2010). Expanding the concept of chemically programmable antibodies to RNA aptamers: chemically programmed biotherapeutics. Angew. Chem. Int. Ed. Engl. 49, 5934-5937.

(20) Dubrovska, A., Kim, C., Elliott, J., Shen, W., Kuo, T.-H., Koo, D.-I., Li, C., Tuntland, T., Chang, J., Groessl, T., et al. (2011). A chemically induced vaccine strategy for prostate cancer. ACS Chem. Biol. 6, 1223-1231.

(21) Sheridan, R.T., Hudon, J., Hank, J.A., Sondel, P.M., and Kiessling, L.L. (2014). Rhamnose glycoconjugates for the recruitment of endogenous anti-carbohydrate antibodies to tumor cells. ChemBioChem 15, 1393-1398.

(22) Rullo, A.F., Fitzgerald, K.J., Muthusamy, V., Liu, M., Yuan, C., Huang, M., Kim, M., Cho, A.E., and Spiegel, D.A. (2016). Reengineering the immune response to metastatic cancer: antibodyrecruiting small molecules targeting the urokinase receptor. Angew. Chem. Int. Ed. Engl. 55, 3642-3646.

(23) Galili, U., Rachmilewitz, E.A., Peleg, A., and Flechner, I. (1984). A unique natural human IgG antibody with anti-alphagalactosyl specificity. J. Exp. Med. 160, 1519-1531.

(24) Parker, W., Bruno, D., Holzknecht, Z.E., and Platt, J.L. (1994) Characterization and affinity isolation of xenoreactive human natural antibodies. J. Immunol. 153, 3791-3803.

(25) Galili, U. (2005). The alpha-gal epitope and the anti-Gal antibody in xenotransplantation and in cancer immunotherapy. Immunol. Cell Biol. 83, 674-686.

(26) Farah, F. (1973). Natural antibodies specific to the 2, 4dinitrophenyl group. Immunology 25, 217-226.

(27) Ortega, E., Kostovetzky, M., and Larralde, C. (1984). Natural DNP-binding immunoglobulins and antibody multispecificity. Mol. Immunol. 21, 883-888.

(28) Mitoma, H., Horiuchi, T., Tsukamoto, H., Tamimoto, Y., Kimoto, Y., Uchino, A., To, K., Harashima, S.I., Hatta, N., and Harada, M. (2008). Mechanisms for cytotoxic effects of antitumor necrosis factor agents on transmembrane tumor necrosis factor $\alpha$-expressing cells: comparison among infliximab, etanercept, and adalimumab. Arthritis Rheum. 58, 1248-1257.

(29) Vazquez-Lombardi, R., Loetsch, C., Zinkl, D., Jackson, J., Schofield, P., Deenick, E.K., King, C., Phan, T.G., Webster, K.E., Sprent, J., et al. (2017). Potent antitumour activity of interleukin-2-Fc fusion proteins requires $\mathrm{Fc}$-mediated depletion of regulatory T-cells. Nat. Commun. $8,15373$.

(30) Dai, H.-S., Griffin, N., Bolyard, C., Mao, H.C., Zhang, J., Cripe, T.P., Suenaga, T., Arase, H., Nakano, I., Chiocca, E., et al. (2017). The Fc domain of immunoglobulin is sufficient to bridge NK cells with virally infected cells. Immunity 47, 159-170.e10.

(31) Sasaki, K., Miyashita, Y., Asai, D., Funamoto, D., Sato, K., Yamaguchi, Y., Mishima, Y., Iino, T., Takaishi, S., Nagano, J., et al. (2018). A peptide inhibitor of antibody-dependent cellmediated cytotoxicity against EGFR/folate receptor- $\alpha$ double positive cells. MedChemComm 9, 783-788. 
(32) Li, B., Zhao, L., Guo, H., Wang, C., Zhang, X., Wu, L., Chen, L., Tong, Q., Qian, W., Wang, H., et al. (2009). Characterization of a rituximab variant with potent antitumor activity against rituximab-resistant B-cell lymphoma. Blood 114, 5007-5015.

(33) Cartron, G., Dacheux, L., Salles, G., Solal-Celigny, P., Bardos, P., Colombat, P., and Watier, H. (2002). Therapeutic activity of humanized anti-CD20 monoclonal antibody and polymorphism in IgG Fc receptor FcyRIIIa gene. Blood 99, 754-758.

(34) Gong, Y., Zhang, L., Li, J., Feng, S., and Deng, H. (2016). Development of the double cyclic peptide ligand for antibody purification and protein detection. Bioconjug. Chem. 27, 1569-1573.

(35) Chen, C., Ke, J., Zhou, X.E., Yi, W., Brunzelle, J.S., Li, J., Yong, E.-L., Xu, H.E., and Melcher, K. (2013). Structural basis for molecular recognition of folic acid by folate receptors. Nature 500, 486-489.

(36) Sega, E.I., and Low, P.S. (2008). Tumor detection using folate receptor-targeted imaging agents. Cancer Metastasis Rev. 27, 655-664.

(37) Douglass Jr, E.F., Miller, C.J., Sparer, G., Shapiro, H., and Spiegel, D.A. (2013). A comprehensive mathematical model for three-body binding equilibria. J. Am. Chem. Soc. 135, 60926099.

(38) Barth, M.J., Mavis, C., Czuczman, M.S., and HernandezIlizaliturri, F.J. (2015). Ofatumumab exhibits enhanced in vitro and in vivo activity compared to rituximab in preclinical models of mantle cell lymphoma. Clin. Cancer Res. 21, 4391-4397.

(39) Mishima, Y., Terui, Y., Mishima, Y., Kuniyoshi, R., Matsusaka, S., Mikuniya, M., Kojima, K., and Hatake, K. (2012). High reproducible ADCC analysis revealed a competitive relation between ADCC and CDC and differences between Fc $\gamma$ Rllla polymorphism. Int. Immunol. 24, 477-483.

(40) Alter, G., Malenfant, J.M., and Altfeld, M. (2004). CD107a as a functional marker for the identification of natural killer cell activity. J. Immunol. Methods 294, 15-22.

(41) Andersen, J.T., Daba, M.B., Berntzen, G., Michaelsen, T.E., and Sandlie, I. (2010). Cross-species binding analyses of mouse and human neonatal $\mathrm{Fc}$ receptor show dramatic differences in immunoglobulin $\mathrm{G}$ and albumin binding. J. Biol. Chem. 285, 48264836.

(42) Gonzalez-Quintela, A., Alende, R., Gude, F., Campos, J., Rey, J., Meijide, L., Fernandez-Merino, C., and Vidal, C. (2008). Serum levels of immunoglobulins (IgG, $\operatorname{IgA}, \operatorname{IgM})$ in a general adult population and their relationship with alcohol consumption, smoking and common metabolic abnormalities. Clin. Exp. Immunol. 151, 42-50.

(43) Overdijk, M.B., Verploegen, S., Ortiz Buijsse, A., Vink, T., Leusen, J.H., Bleeker, W.K., and Parren, P.W. (2012). Crosstalk between human IgG isotypes and murine effector cells. J. Immunol. 189, 3430-3438.

(44) Petkova, S.B., Akilesh, S., Sproule, T.J., Christianson, G.J., Al Khabbaz, H., Brown, A.C., Presta, L.G., Meng, Y.G., Roopenian, D.C. (2006). Enhanced half-life of genetically engineered human IgG1 antibodies in a humanized FcRn mouse model: potential application in humorally mediated autoimmune disease. Int. Immunol. 18, 1759-1769.

(45) Jung, Y., Kang, H.J., Lee, J.M., Jung, S.O., Yun, W.S., Chung, S.J., and Chung, B.H. (2008). Controlled antibody immobilization onto immunoanalytical platforms by synthetic peptide. Anal. Biochem. 374, 99-105.

(46) French, M., and Harrison, G. (1984). Serum IgG subclass concentrations in healthy adults: a study using monoclonal antisera. Clin. Exp. Immunol. 56, 473-475.

(47) Klouche, M., Bradwell, A.R., Wilhelm, D., and Kirchner, H. (1995). Subclass typing of IgG paraproteins by immunofixation electrophoresis. Clin. Chem. 41, 1475-1479.

(48) Brüggemann, M., Williams, G.T., Bindon, C.I., Clark, M.R., Walker, M.R., Jefferis, R., Waldmann, H., and Neuberger, M.S (1987). Comparison of the effector functions of human immunoglobulins using a matched set of chimeric antibodies. J. Exp. Med. 166, 1351-1361.

(49) Buss, N.A., Henderson, S.J., McFarlane, M., Shenton, J.M., and De Haan, L. (2012). Monoclonal antibody therapeutics: history and future. Curr. Opin. Pharmacol. 12, 615-622.

(50) Shields, R.L., Lai, J., Keck, R., O'Connell, L.Y., Hong, K., Meng, Y.G., Weikert, S.H., and Presta, L.G. (2002). Lack of fucose on human IgG1 N-linked oligosaccharide improves binding to human FcyRIII and antibody-dependent cellular toxicity. J. Biol. Chem. 277, 26733-26740.

(51) Xiao, H., Woods, E.C., Vukojicic, P., and Bertozzi, C.R. (2016). Precision glycocalyx editing as a strategy for cancer immunotherapy. Proc. Natl. Acad. Sci. U.S.A. 113, 10304-10309.

(52) Pagan, J.D., Kitaoka, M., and Anthony, R.M. (2018). Engineered sialylation of pathogenic antibodies in vivo attenuates autoimmune disease. Cell 172, 564-577. e13. 